Virginia Commonwealth University

VCU Scholars Compass

2010

\title{
Dual-doped thermographic phosphor particles as surrogates for green fluorescent protein-labeled cells in tests of cytometric neurocatheters
}

\author{
S. W. Allison \\ Oak Ridge National Laboratory, allisonsw@ornl.gov \\ G. T. Gillies \\ Virginia Commonwealth University, University of Virginia
}

Follow this and additional works at: http://scholarscompass.vcu.edu/neurosur_pubs

Part of the Medicine and Health Sciences Commons

Allison, S. W., and Gillies, G. T. Dual-doped thermographic phosphor particles as surrogates for green fluorescent protein-labeled cells in tests of cytometric neurocatheters. Journal of Applied Physics, 107, 064904 (2010). Copyright (C) 2010 American Institute of Physics.

\section{Downloaded from}

http://scholarscompass.vcu.edu/neurosur_pubs/5

This Article is brought to you for free and open access by the Dept. of Neurosurgery at VCU Scholars Compass. It has been accepted for inclusion in Neurosurgery Publications by an authorized administrator of VCU Scholars Compass. For more information, please contact libcompass@vcu.edu. 


\title{
Dual-doped thermographic phosphor particles as surrogates for green fluorescent protein-labeled cells in tests of cytometric neurocatheters
}

\author{
S. W. Allison ${ }^{1, a)}$ and G. T. Gillies ${ }^{2}$ \\ ${ }^{1}$ Sensors and Controls Research Group, Oak Ridge National Laboratory, Oak Ridge, Tennessee 37831 - \\ 6054, USA \\ ${ }^{2}$ Department of Mechanical and Aerospace Engineering, University of Virginia, Charlottesville, Virginia \\ 22904-4746, USA and Department of Neurosurgery, Virginia Commonwealth University, Richmond, \\ Virginia 23298-0631, USA
}

(Received 11 December 2009; accepted 7 February 2010; published online 30 March 2010)

\begin{abstract}
We investigated the laser-induced fluorescence of particles of a compound thermographic phosphor $\mathrm{La}_{2} \mathrm{O}_{2} \mathrm{~S}: \mathrm{Eu}(1 \%)$ and $\mathrm{Gd}_{2} \mathrm{O}_{2} \mathrm{~S}: \mathrm{Eu}(1 \%)$ to see if they can serve as a surrogate for cells transfected with the green fluorescent protein, in tests of neurocatheters used for intraparenchymal cell delivery. At an excitation wavelength of $337 \mathrm{~nm}$ and a concentration of $\approx 2 \times 10^{6}$ particles $\mathrm{ml}^{-1}$, the resulting slurries produced fluorescence intensities at $625 \mathrm{~nm}$ that were within a factor of 2 of those produced by similar number densities of relevant cells, thus suggesting the utility of this approach. (C) 2010 American Institute of Physics. [doi:10.1063/1.3359656]
\end{abstract}

\section{INTRODUCTION}

We are developing a novel cell delivery catheter that is capable of in situ flow cytometry, ${ }^{1,2}$ for eventual clinical use in the treatment of Parkinson disease. ${ }^{3,4}$ In such therapies, delivery into the brain of too few viable cells constitutes an ineffective dose, while delivery of too many results in excessive competition for nutrients and oxygen. Hence, the goal is to confirm the intraoperative delivery of the appropriate number of cells to optimize the efficacy of the procedure.

Several technical steps are needed to bring such a device to productive clinical testing. For instance, optical fibers will be used to photonically monitor the cells as they pass through the port hole of the catheter. Specifically, they must deliver light to the cells, collect the resulting cellular fluorescence, and then convey it to an appropriate detector in the monitoring system. It is important to determine the best configuration of the optical fibers within the tip of the catheter for this purpose, with the resulting design(s) tested initially using inexpensive and readily available in vitro models of positive pressure infusion, ${ }^{5,6}$ which is a method for intracranial cell delivery. In some of our preliminary studies, green fluorescent protein (GFP)-transfected RT2 cells ${ }^{7}$ were used as luminescent cellular beacons in suspensions that were pumped through the port hole of an early prototype of the catheter, for purposes of testing the feasibility of this approach to cytometric delivery. This has been a useful method because it involves living cells and thus mimics a realistic clinical situation. However, the complexities and expense of experiments involving viable cells argues for the introduction of an inexpensive surrogate that would be easier to use in routine testing of catheter design details, noncritical performance studies, etc. Cell-sized particles of thermographic phosphors are candidates for such a surrogate because of some cost and performance advantages over, e.g., fluorescent

\footnotetext{
a) Author to whom correspondence should be addressed. Electronic mail: allisonsw@ornl.gov.
}

polymeric microbeads. In particular, such materials exhibit a well-characterized temperature dependence in their fluorescence decay lifetimes, and thus offer the additional possibility of in situ thermometry, in those cases where such a diagnostic would be useful. Therefore, we investigated the laserinduced fluorescence of slurries of phosphor particles that were pumped through the distal tip side port hole of the same catheter through which actual cell slurries had been pumped, to validate the utility of such particles as surrogates for GFPlabeled cells.

\section{BACKGROUND}

The term thermographic phosphor describes a large class of oxides and oxysulfides, the fluorescence of which is activated by inclusion of one or more rare-earth dopants. We have studied many different types of applications for these materials,${ }^{8}$ including their use as flow tracers in simulations of positive pressure infusion for the treatment of brain tumors ${ }^{9}$ and their performance in nanoscale fluorescence thermometry at physiological temperatures. ${ }^{10}$ With respect to the present study, the key feature of such materials is the particle luminescence. To serve as a useful surrogate for viable cells in our cytometric catheter, the particles must exhibit measurable fluorescence when passing through the excitation optical field that interrogates the port hole. The results of many previous studies ${ }^{8-10}$ suggested that the fluorescence signal strengths from any number of different candidate phosphors should be suitable for this purpose. Therefore, the choice of the particular material to be used was largely a question of other considerations, such as particle size, cost of material, and availability. The mean diameter of a postnatal, postmortem human neural progenitor cell (hNPC) is known ${ }^{11}$ to be within the range of 5-10 $\mu \mathrm{m}$. Specific variances within these dimensions will depend on the actual type and source of cells, the method of culture, handling techniques, etc. Moreover, the cells also tend to clump together in media, thus further complicating the ques- 
tion of mean diameter and necessitating sonication of the suspensions to keep the cells separated. Likewise, particles of most thermographic phosphors will agglomerate under various conditions but aspirative mixing can minimize the problem. At the micrometer scale the as-supplied phosphors have typically been sifted through fine mesh screens to set a maximum particle size for a particular batch. Our candidate phosphor was a 50:50 mix of $\mathrm{La}_{2} \mathrm{O}_{2} \mathrm{~S}: \mathrm{Eu}(1 \%)$ and $\mathrm{Gd}_{2} \mathrm{O}_{2} \mathrm{~S}: \mathrm{Eu}(1 \%)$ (Phosphor Technology Ltd., Stevenage, U.K.). As per the manufacturer, the $\mathrm{La}_{2} \mathrm{O}_{2} \mathrm{~S}: \mathrm{Eu}$ component had a mean particle size of $8.6 \mu \mathrm{m}$ at a volume percentage of $95 \%$ (i.e., $95 \%$ of the particles were $8.6 \mu \mathrm{m}$ or smaller), and $4.2 \mu \mathrm{m}$ at a volume percentage of $50 \%$. Likewise, the $\mathrm{Gd}_{2} \mathrm{O}_{2} \mathrm{~S}$ : Eu component had a mean particle size of $7.9 \mu \mathrm{m}$ at a volume percentage of $95 \%$ and $4.0 \mu \mathrm{m}$ at a volume percentage of $50 \%$. The resulting overlap in the nominal size ranges of the phosphor particles and the hNPC cells was deemed sufficient for our purposes.

The fluorescence spectrum of compound phosphors of this kind can be more complex than that of single-component phosphors (such as $\mathrm{La}_{2} \mathrm{O}_{2} \mathrm{~S}$ : Eu by itself). However, we have previously made photospectrometric measurements of such materials $^{8,12}$ and thus further reason for choosing one here. In fact, the emission characteristics have been studied in detail in other contexts, ${ }^{8,13}$ and it is known that the positions of the spectral lines are essentially identical in both components of this phosphor. The luminescence is easily distinguishable from any spurious or stray light that might be present in the experimental arrangement, and the fluorescence of this phosphor does not quench when the particles are submerged in aqueous media. This latter point is important here because a liquefied suspension of the particles must be pumped through the catheter under test in order to properly simulate the cell delivery process. Finally, the broad emission above $600 \mathrm{~nm}$ originates from ${ }^{5} \mathrm{D}_{0}$ states and is temperature-independent for both phosphor components. ${ }^{14}$ Future work could exploit the temperature dependence of ${ }^{5} \mathrm{D}_{2}$ emission, typically below about $520 \mathrm{~nm}$, which is most striking for the lanthanum phosphor. It is distinguishable from the gadolinium material by a much shorter decay time. Thus, potential exists for simultaneous determination of particle concentration and temperature.

\section{EXPERIMENTAL ARRANGEMENT}

In preparation for use, a volume of roughly $100 \mathrm{~mm}^{3}$ of the dual-doped phosphor was mixed into approximately $5 \mathrm{ml}$ of Triton X-100 (SPI-Chem, West Chester, PA) nonionic surfactant solution and $45 \mathrm{ml}$ of water, to form $50 \mathrm{ml}$ of suspension. The surfactant was used to help prevent clumping of the particles. The suspension was stirred thoroughly and then aspiratively mixed within a polyethylene suction pipette from which it could then be passed into the catheter via a Luer port.

The catheter under test was a modified version of the inner tube of a NexGen Medical Systems, Inc. (Reno, NV) CNS-I acute neurological catheter, as described elsewhere by Fillmore et al. ${ }^{3}$ The modification consisted of mounting two optical fibers (with a nominal diameter of $100 \mu \mathrm{m}$ each)

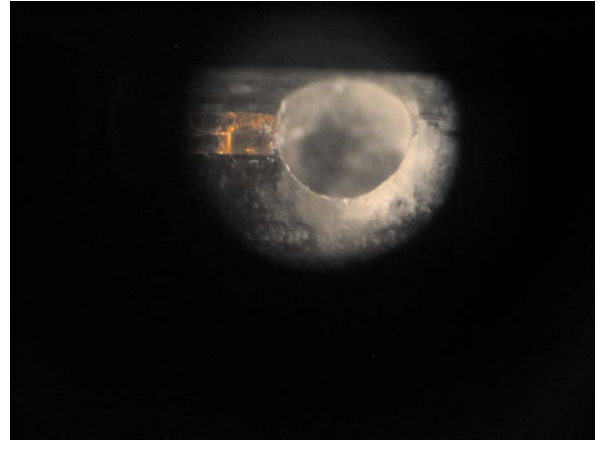

FIG. 1. (Color online) Photomicrograph of the port hole at distal tip of catheter, with a pair of $100 \mu \mathrm{m}$ diameter optical fibers positioned just at the left edge. The port hole is approximately $1 \mathrm{~mm}$ in diameter.

onto the outer surface of the catheter body such that the tips of the optical fibers were positioned against the proximal edge of the catheter's side port hole, as shown in the photomicrograph of Fig. 1. A simple ray trace in relation to the diameter of the port hole is shown to scale in Fig. 2. One optical fiber delivers light over a portion of the surface area of the port hole, and that light emerges at a characteristic angle of expansion usually denoted as the numerical aperture (NA). For these optical fibers, NA $=0.22$, corresponding to a half-angle of about $13^{\circ}$. The diameter of the optical fiber's core is $100 \mu \mathrm{m}$, with a cladding diameter of $110 \mu \mathrm{m}$, and a polyimide jacket (the golden-colored material seen in the color version of Fig. 1) that yields a final outer diameter of $\approx 120 \mu \mathrm{m}$. With reference to Fig. 2, let the upper optical fiber deliver the laser light, the conical emergence of which is denoted by the light- and medium-shaded regions, 1 and 2, respectively, of the figure (the yellow and blue regions in the color version of the figure). The bottom optical fiber then acts as the receiver, and it conveys the cellular fluorescence signals to an attached detector. It views the medium- and dark-shaded regions, 2 and 3, respectively, (the blue and green regions in the color version of the figure). To be detected, a fluorescent particle or GFP-labeled cell must be within the illumination zone of the top optical fiber and the acceptance zone of the bottom one. The overlap of these two regions constitutes the medium-shaded (blue) zone, 2, shown

\section{Two Fiber Probe}

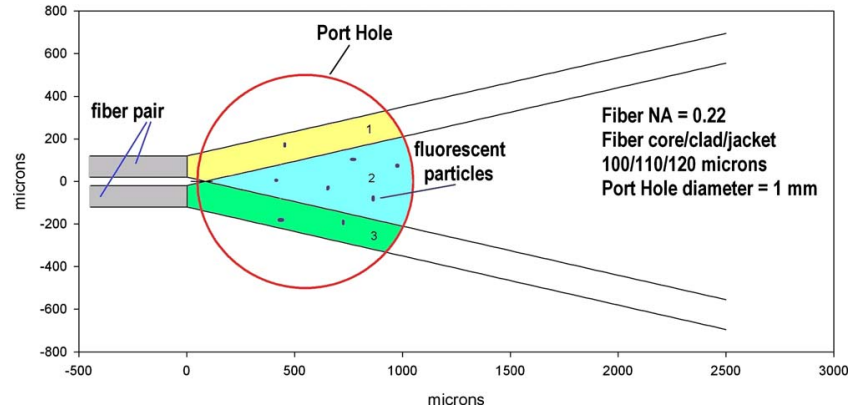

FIG. 2. (Color online) Schematic diagram of fiber illumination and collection profiles. The light from the illumination fiber fills the light- and medium-shaded (yellow and blue) zones, 1 and 2. The receiver fiber views the medium- and dark-shaded (blue and green) zones, 2 and 3. The mediumshaded (blue) zone, 2, shows the region in which the fluorescent particles will be both illuminated and detected. 
in Fig. 2. In the present catheter design, $\approx 1 / 3$ of the port hole's surface area was sampled because a relatively small optical fiber size was chosen in order to keep the profile of the outer wall of the catheter as smooth as possible. Modifications of the arrangement that enable sampling the full surface area are described elsewhere. ${ }^{16}$

To detect the fluorescence of the phosphor particles that were passing through the catheter's port hole, we used a high sensitivity photomultiplier tube (PMT) to monitor the optical signal that was conveyed within the return fiber. Also, for inspection purposes, a Sony SSC-C374 CCD color video camera with an InfiniVarTM video microscope attachment was used for visual observation of the flow through and the fluorescence within the port hole of the catheter. To drive the fluorescence, a train of uv pulses from a VSL-337ND laser (wavelength $=337 \mathrm{~nm}$ ) was conveyed to the port hole through the delivery optical fiber. The PMT output signal was acquired by a high speed digitizing oscilloscope from which the data could be downloaded to a laboratory computer for offline analysis. The catheter under test was mounted on a precision laboratory jack to aid in proper positioning of it relative to the video microscope. The optical fibers were coupled to the laser output port and the PMT inlet port via Sub Multi Assembly (SMA) connectors. Careful alignment of the components was critical, in part because of the small apertures at the fiber ends (nominally $100 \mu \mathrm{m}$ in diameter). The entire measurement system was situated on a large surface area optical table for mechanical stability, convenience of alignment, etc.

During the experiments, the pipette containing the phosphor suspension was inserted into the catheter's Luer port, and its bulb was compressed manually to initiate flow of the suspension through the lumen. Control measurements using only water as the surrogate cell suspension were also carried out, with the catheter lumen flushed and dried between all consecutive runs. In each of the experiments, the video microscope image was displayed on a high resolution monitor to identify fluorescence or the lack thereof, and thus corroborate that the PMT signals arose from the target luminescence. All data were taken with the laboratory darkened to reduce noise and minimize the detector's background level.

\section{RESULTS}

At low pulse rates $(\leq 20 \mathrm{~Hz})$, the average power of the laser was $\approx 6 \mathrm{~mW}$, with $300 \mu \mathrm{J}$ per pulse. ${ }^{15}$ At these levels of illumination and with the phosphor suspension just filling the distal tip of the catheter but not flowing out of the port hole (i.e., not dripping from it), fluorescence signals such as those shown in Fig. 3(a) were obtained routinely. As seen there, test no. 1 produced a peak signal of roughly $200 \mathrm{mV}$ while that of test no. 2 was somewhat smaller, roughly 150 $\mathrm{mV}$, most likely due to a slight shift in the very sensitive alignment of the fibers at the edge of the port hole. On the other hand, the control measurements showed no signal resolvable above the background. Figure 3(b) shows the emission spectrum of the phosphor obtained with an Ocean Optics (Dunedin, Florida) model USB2000 FLG minispectrometer.
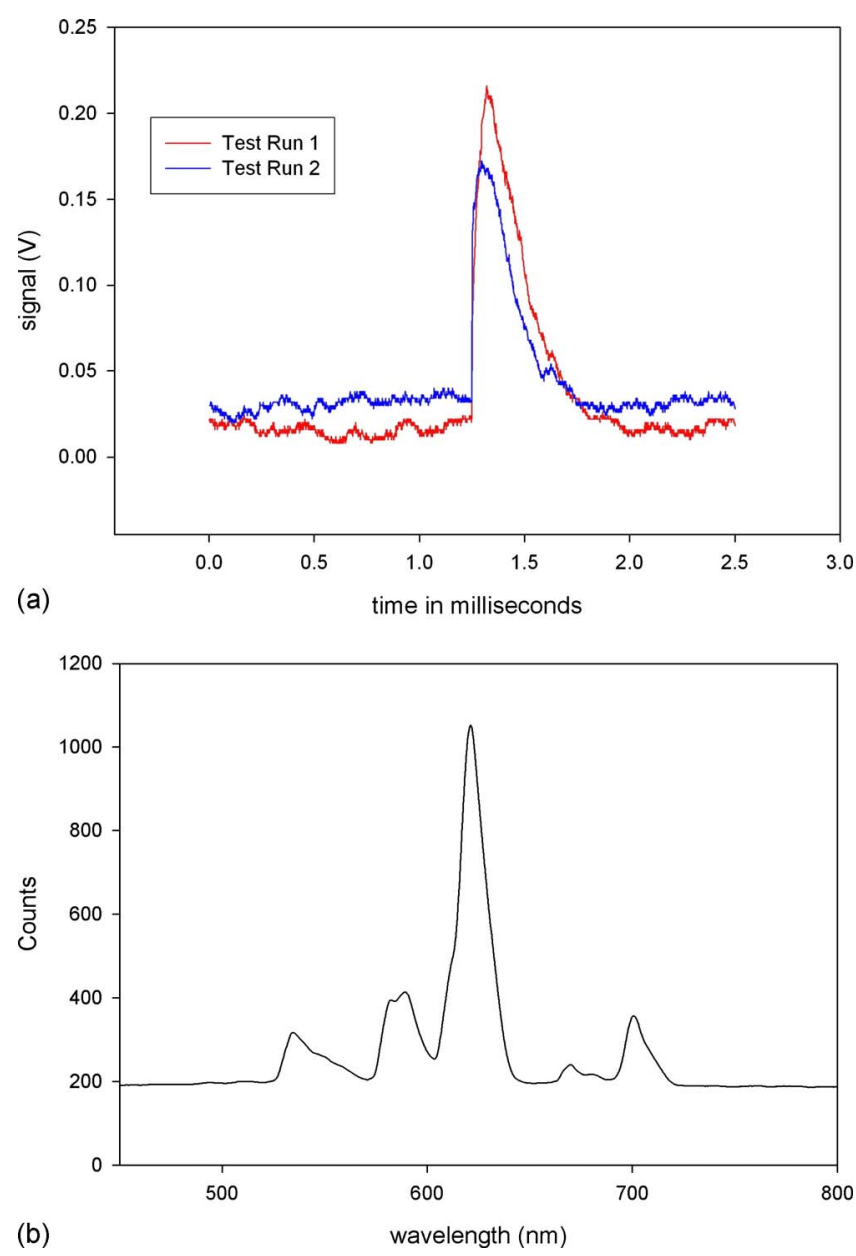

FIG. 3. (Color online) (a) The fluorescence decay curves from two different measurements made on a suspension of $\mathrm{La}_{2} \mathrm{O}_{2} \mathrm{~S}: \mathrm{Eu}(1 \%), \mathrm{Gd}_{2} \mathrm{O}_{2} \mathrm{~S}: \mathrm{Eu}(1 \%)$. (b) The fluorescence spectrum for this material showing a dominant peak at $\approx 625 \mathrm{~nm}$.

In earlier (unpublished) experiments with the GFPtransfected RT2 cells, we found that the same experimental arrangement had a sensitivity of $\approx 7 \mathrm{mV}$ per fluorescent cell. The number of such cells that would produce the larger of the signals shown in Fig. 3(a) would thus be $(200 \mathrm{mV}) /(7$ $\mathrm{mV}$ ), or about 30 cells. In what follows, we make an orderof-magnitude estimate of the number of fluorescing phosphor particles needed to produce this $200 \mathrm{mV}$ signal, to see how it compares against this number of cells.

As mentioned above, $\approx 100 \mathrm{~mm}^{3}$ of phosphor was used to create the $50 \mathrm{ml}$ suspension. Given a particle diameter rounded-up to $10 \mu \mathrm{m}$ and an assumed packing fraction of 0.5 , we estimate that there were on the order of $10^{8}$ particles in the sample used to create the suspension, i.e., 2 $\times 10^{6}$ particles $\mathrm{ml}^{-1}\left(=2 \times 10^{3}\right.$ particles $\left.\mu \mathrm{l}^{-1}\right)$ within the 50 $\mathrm{ml}$ volume. The diameter of the port hole was $1 \mathrm{~mm}$. The surface area of the port hole times the inner diameter of the catheter $(1.2 \mathrm{~mm})$ thus yields an estimate on the order of $1 \mu \mathrm{l}$ as the (cylindrical) volume of suspension inside the region bounded geometrically by the structure of the port hole and the inner lumen of the catheter. At the particle density found above, that would mean there were roughly 2,000 phosphor particles within that region. As per Fig. 1, the optical fibers were positioned on the catheter above the lip at 
the edge of the port hole. The resulting viewing angle would thus permit coverage of (1) the meniscus layer of the suspension above the surface of the port hole and (2) that part of the interior region below the surface of the port hole accessible within the NA of the fiber. We estimate that this interrogation zone constituted perhaps a percent of the $\approx 1 \mu$ l volume of the particle suspension contained beneath the port hole of the catheter. Hence, there would have been about 20 particles within it. This is within a factor of 2 of the number of cells $(\approx 30)$ that were needed to produce the corresponding signal, as noted above. Of course, if the fluorescence intensity per cell were substantially weaker than that per particle, this ratio would change by the appropriate numerical factor. In any case, this result does demonstrate that the low-density suspension of phosphor particles used here has photo-optical characteristics that were similar enough to those of the GFPlabeled cells to warrant further investigation and use in our application.

\section{DISCUSSION AND CONCLUSIONS}

As noted above, the alignment of the optical fibers appeared to be a critical factor in these studies. This can be understood by inspection of Fig. 2, the area of the overlap region, 2, clearly depends on not only on how parallel the fibers are but also the relative distance between the emitter and receiver optical fibers. Even though the distal tips of the fibers were glued in place with cyanoacrylate next to the edge of the port hole, the bonds were still relatively delicate and it is not surprising that handling of the catheter between experiments would lead to the observed differences in signal strength. To overcome any such problem, we have redesigned the distal tip of the catheter. ${ }^{16}$ In this new version of it, the tip has channels in which the optical fibers are rigidly embedded, and beam steering via counter-positioned optical fiber stubs with ends polished at $45^{\circ}$ angles is used to convey the illumination signals across the surface area of the port hole. Preliminary versions of it have been built and tested, and the results are reported elsewhere. ${ }^{2}$

Moreover, there are several other approaches to designing a configuration of optical fibers that will sample the entire area of the port hole rather than just a conical fraction of it as was done here, and thus significantly increase the size of the working signal. For instance, more optical fibers could be added, but at the expense of increasing the roughness of the surface profile of the catheter. If this were impractical, the optical fiber pair in the original design might be retracted from the edge of the port hole to a distance where the region of overlap expanded to include the entire region of interest. In this case, a beam homogenizer placed at the optical fiber terminus could serve to contain and homogenize both the delivery and receiving profiles. ${ }^{17}$ One such suitable device would be a rectangular prism of sufficient length. Moreover, there is another reason to consider a homogenizer. Since the light emerging from the optical fiber is characterized by a Gaussian distribution, the acceptance profile can be as well.

In the preliminary experiments reported here, we followed a conservative protocol that was applied uniformly to all measurements, in which the chemistry and mixing of each sample was carried out as per best estimates of how the suspension should be prepared. This was done because it was outside the scope of the present feasibility study to make a full range of detailed measurements of signal strength versus particle concentration in the suspension, at all of the concentrations that would be of interest in the cell suspensions. However, measurements of that kind are planned for future work. In particular, aliquot ratios similar to those in our cell studies will be prepared in which not only the number density of particles but also the volume percentage of the surfactant will be varied. The limiting cases, of course, would be for measurements made on water with no particles (i.e., $0 \%$ concentration, as a null signal control) and similarly on a fluidless film of the phosphor (i.e., $100 \%$ concentration, to provide the upper bound on signal strength). Further tests in this series will include measurements in which the variables of interest are (a) mixing time, (b) settling time, and (c) temperature of the suspension. Gaining an empirical understanding of these factors will be important in clarifying quantitatively how the degree of particle agglomeration affects signal strength. The results from those studies will also be compared against our cell measurements in order to further validate the usefulness of thermographic phosphors in this application.

In conclusion, slurries containing particles of thermographic phosphors can serve as surrogates for fluorescent cell suspensions in studies aimed at developing in situ flow cytometry capabilities in neurocatheters. Our data have shown that the similarities in particle size and fluorescence characteristics warrant their use for this purpose.

\section{ACKNOWLEDGMENTS}

The authors thank their colleagues B. M. Evans, S. M. Goedeke and M. R. Cates (ORNL) and H. L. Fillmore and W. C. Broaddus, M.D. (VCU) for their interest in this work and for several useful discussions. Research sponsored in part by the Laboratory Directed Research and Development Program of Oak Ridge National Laboratory (ORNL), managed by UT-Battelle, LLC for the U.S. Department of Energy under Contract No. DE-AC05-00OR22725. The work at the University of Virginia was funded in part by the Kopf Family Foundation, Inc. and the Royalty Distribution Program of the University of Virginia Patent Foundation.

${ }^{1}$ J. Kucharczyk, G. T. Gillies, W. C. Broaddus, and H. Fillmore, U.S. Patent Application Publication No. US 2003/0204171 A1, 30 October 2003.

${ }^{2}$ B. M. Evans III, S. W. Allison, H. L. Fillmore, W. C. Broaddus, R. L. Dyer, and G. T. Gillies, "Cytometric catheter for neurosurgical applications," J. Med. Eng. Technol. (to be published).

${ }^{3}$ H. L. Fillmore, K. L. Holloway, and G. T. Gillies, Neurorehabilitation 20, 233 (2005).

${ }^{4}$ K. Serpersu, E. C. Bowman, J. A. Watson, B. M. Evans III, G. T. Gillies, and H. L. Fillmore, Conference Proceeding of IEEE on BSEC 2009, edited by B. M. Evans III, (Oak Ridge National Laboratory, Oak Ridge, Tennessee, 2009).

${ }^{5}$ M. A. Bauman, G. T. Gillies, R. Raghavan, M. L. Brady, and C. Pedain, Nanotechnology 15, 92 (2004).

${ }^{6}$ Z.-J. Chen, G. T. Gillies, W. C. Broaddus, S. S. Prabhu, H. L. Fillmore, R. M. Mitchell, F. D. Corwin, and P. P. Fatouros, J. Neurosurg. 101, 314 (2004).

${ }^{7}$ H. L. Fillmore, J. Shurm, P. Furqueron, S. S. Prabhu, G. T. Gillies, and W. C. Broaddus, Cancer Lett. 141, 9 (1999). 
${ }^{8}$ S. W. Allison and G. T. Gillies, Rev. Sci. Instrum. 68, 2615 (1997).

${ }^{9}$ G. T. Gillies, S. W. Allison, and B. M. Tissue, Nanotechnology 13, 484 (2002).

${ }^{10}$ S. W. Allison, G. T. Gillies, A. J. Rondinone, and M. R. Cates, Nanotechnology 14, 859 (2003).

${ }^{11}$ P. H. Schwartz, "National Human Neural Stem Cell Resource," http:// www.nhnscr.org (accessed November 18, 2009).

${ }^{12}$ S. W. Allison, L. A. Boatner, and G. T. Gillies, Appl. Opt. 34, 5624 (1995).

${ }^{13}$ S. W. Allison, M. R. Cates, and G. T. Gillies, Rev. Sci. Instrum. 73, 1832
(2002).

${ }^{14}$ C. W. Struck and W. H. Fonger, J. Lumin. 1-2, 456 (1970).

15“"VSL-337ND-S Nitrogen Laser," Spectra-Physics Division (Newport Corporation, Santa Clara, California). http://www.newport.com/store/ genproduct.aspx $\mathrm{id}=368232 \&$ lang $=1033 \&$ Section $=$ Spec $\quad($ accessed November 18,2009 ).

${ }^{16}$ G. T. Gillies, H. L. Fillmore, W. C. Broaddus, B. M. Evans III, and S. W. Allison, International Patent Application Publication No. WO 2008/ 057370 A2, 15 May, 2008.

${ }^{17}$ S. W. Allison and G. T. Gillies, Appl. Opt. 33, 1802 (1994). 